\section{CAPTURING A CLEAR IMAGE}

Evident's dental imaging products include the Cammy, a lightweight intraoral camera. Capturing a clear image of your patient's mouth, the advanced Cammy instantly enables the patient to see exactly what you see.

In addition, its distinctive mini-head design enables you to access even the hardest to reach areas, helping identify cracks and find root canals. Capturing images of your patients' mouth can be achieved with the zoom toggle and freeze buttons. Connecting directly to your computer via a USB port, these images can be easily stored, retrieved and transmitted.

The easy to use, affordable digital dental radiography system Digirex uses state-of-the-art CCD technology to provide you with high definition imaging

\section{DIGITAL IMAGING OFFER}

The Dental Directory latest promotion on digital imaging systems offers readers the chance to get a free intraoral camera.

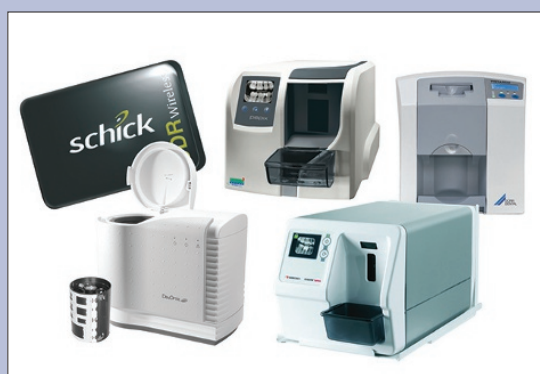

for perfect X-rays, enabling faster diagnosis and eliminating the cost of chemicals and film.

Simply connect Digirex to your computer via a USB port and you can immediately begin to store, retrieve and transmit images electronically and even adjust or magnify images for optimum viewing.

\section{Reader response number 54}

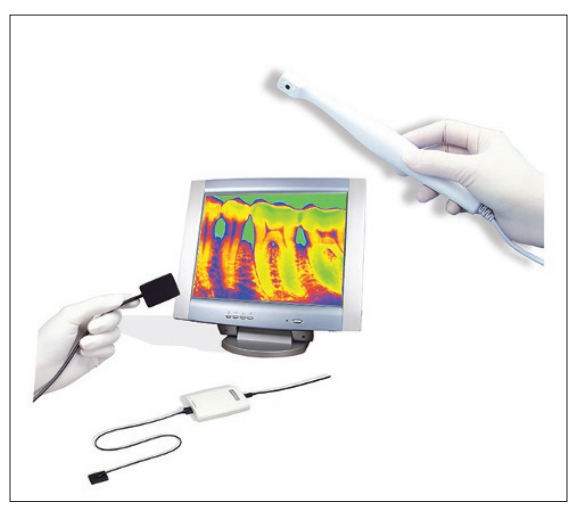

This offer is available when you order either the Schick CDR System, the Digora Optime, the Gendex Den0ptix QST Pan Plus, the Sopro PS Pix or the new Dürr Vistascan Mini Plus. Each of these imaging systems offers state-of-the-art technology and superb image quality, and you will get a free Iopix advanced intraoral camera worth $£ 1,595$.

The Dental Directory experts are available to give you impartial advice on which system best suits your requirements and also have access to very attractive finance options. Reader response number 55

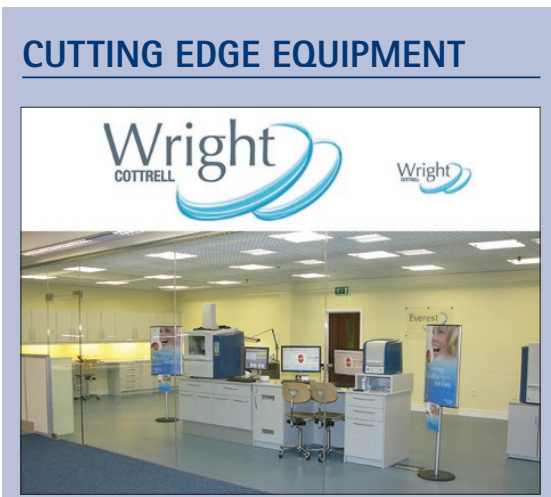

UK dental supplier Wright Cottrell supplies a range of quality products for all indications, including cutting edge equipment such as the Kavo Everest CAD/CAM system.

A complete processing system for strong and aesthetic solutions for all ceramic restorations, the $\mathrm{KaVo}$ Everest CAD/CAM system is an example of holistic design in dental laboratory technology.

The Everest system provides an optimum solution for the demands of contemporary processing, combining advanced technology with maximum economy and quality. The system comprises four components: the Everest scan pro, a measuring unit with exact scanning of the models, the Everest engine, a milling and grinding unit for high precision and automated processing, the Everest Therm, a sintering unit for perfect sintering, and Everest Elements, 12 materials that cover all physical, chemical and biocompatibility requirements of modern dentistry.

Reader response number 57

\title{
INTERACTIVE TREATMENT PROCEDURE
}

Castellini's Videorcam provides illumination for the operating area. Complete with LED flash technology, it has been developed specifically for clinical observation.

All the images captured by the Videorcam have a variety of applications, providing dentists with a more interactive treatment procedure and assisting patients to understand diagnosis and treatment options.

It uses software available from the Videor System, while flashless technology enhances the clarity of the images taken and the soft touch command is controlled by a dental foot control for increased efficiency. Easily transferable to a PC via a USB 2.0 connection, the images captured possess sharpness and precision.

Performing intrabuccal exams is simplified when using Videorcam's miniaturised head and macro function and it creates high resolution, accurate, natural-coloured images.

Reader response number 56

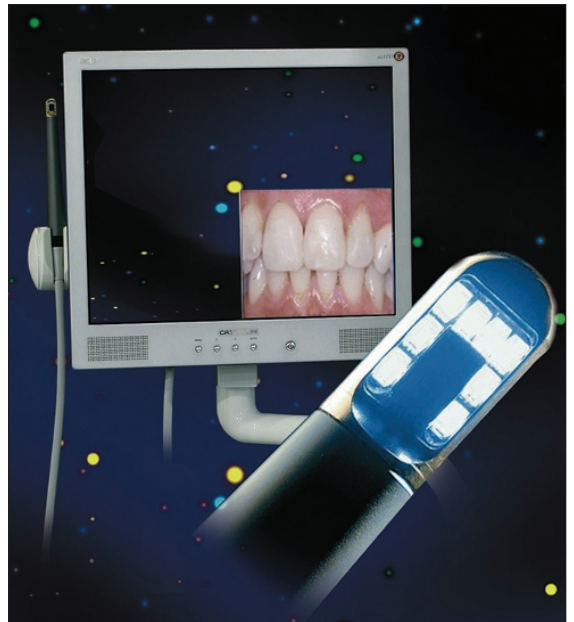

